\title{
Evolution of mud-crack patterns during repeated drying cycles
}

\author{
Lucas Goehring, ${ }^{a b c}$ Rebecca Conroy, ${ }^{a}$ Asad Akhter, ${ }^{a}$ William J. Clegg ${ }^{b}$ and Alexander F. Routh ${ }^{* a c}$
}

\author{
Received 28th October 2009, Accepted 31st March 2010 \\ DOI: 10.1039/b922206e
}

In mud, crack patterns are frequently seen with either an approximately rectilinear or hexagonal tiling. Here we show, experimentally, how a desiccation crack pattern changes from being dominated by $90^{\circ}$ joint angles, to $120^{\circ}$ joint angles. Layers of bentonite clay, a few $\mathrm{mm}$ thick, were repeatedly wetted and dried. When dried, the layers crack. These cracks visibly close when rewetted, but a similar crack pattern forms when the layer is redried, with cracks forming along the lines of previously open cracks. Time-lapse photography was used to show how the sequence in which individual cracks open is different in each generation of drying. The geometry of the crack pattern was observed after each of 25 generations of wetting and drying. The angles between cracks were found to approach $120^{\circ}$, with a relaxation time of approximately 4 generations. This was accompanied by a gradual change in the position of the crack vertices, as the crack pattern evolved. A simple model of crack behavior in a layer where the positions of previously open cracks define lines of weakness is developed to explain these observations.

\section{Introduction}

When slurries dry, they tend to crack. These cracks form sequentially, and when a later, or secondary, crack approaches an earlier, or primary, one it curves to intersect it at $90^{\circ}$, forming a 'T-junction'. The resulting crack pattern divides the dried layer into roughly rectilinear pieces, such as those shown in Fig. 1(a,b). This behavior has been reported in many materials, including clay, ${ }^{1,2}$ colloidal alumina, ${ }^{3}$ corn starch, ${ }^{4-6}$ and coffee grounds. ${ }^{7}$

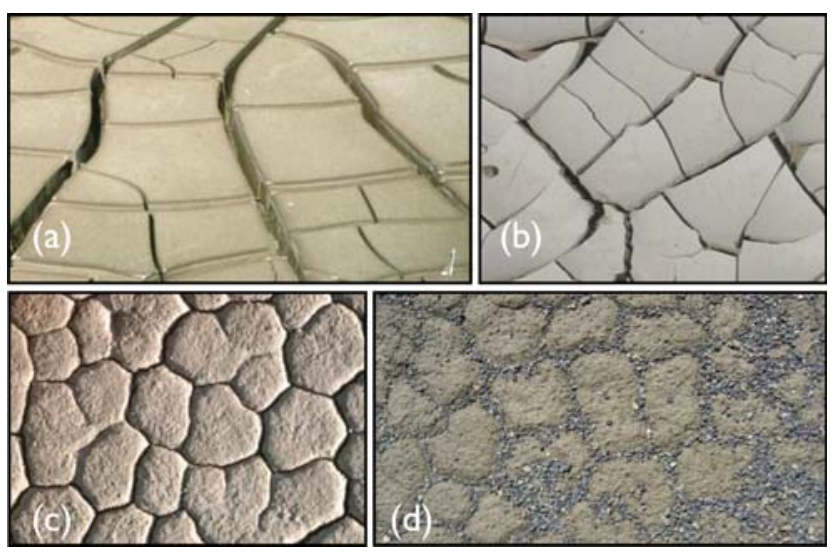

Fig. 1 There are a variety of fracture patterns commonly found in dried mud. (a,b) show rectilinear patterns, where cracks typically meet at 'T-junctions' of $90^{\circ}$ and $180^{\circ}$. (c,d) show, in contrast, hexagonal patterns, where cracks meet at 'Y-junctions' of $120^{\circ}$. [Panels (a,c) courtesy of B. Hallet].

\footnotetext{
${ }^{a}$ Department of Chemical Engineering and Biotechnology, University of Cambridge, Pembroke Street, Cambridge, UK CB2 3RA. E-mail: afr10@cam.ac.uk

${ }^{b}$ Department of Materials Science and Metallurgy, University of Cambridge, Pembroke Street, Cambridge, UK CB2 $3 Q Z$

${ }^{c} B P$ Institute for Multiphase Flow, University of Cambridge, Madingley Rise, Madingley Road, Cambridge, UK CB3 OEZ
}

The patterns found in dried mud are, however, not always as suggested by these experiments. Mud can, as shown in Fig. 1(c,d), contain crack networks that outline roughly hexagonal cells, with cracks intersecting at $120^{\circ}$, forming 'Y-junctions'. The origin of these differences in geometry is not understood.

In this paper, we consider the cracks that form when clay dries. We describe how the junctions between cracks can evolve when the layer is repeatedly wetted and dried, and show how a rectilinear crack pattern changes into a hexagonal crack pattern. These observations are explained using a simple model of crack formation, where the positions of previously open cracks influence the development of the next generation of cracking.

\section{Materials and methods}

Slurries of bentonite clay (Acros Organics, Bentonite K-10) and de-ionized water were prepared by adding water to dry bentonite powder until it could be easily stirred. Slightly less than two parts of water were added for every part bentonite, by mass. These slurries were poured into flat-bottomed polystyrene petri dishes (150 mm diameter, $10 \mathrm{~mm}$ height). At the end of each experiment, the thickness of the clay layers was measured by picking up several dried pieces, and measuring their thickness with micro-callipers.

Dishes were placed approximately $50 \mathrm{~cm}$ below a pair of $150 \mathrm{~W}$ halogen heat lamps, and allowed to dry, and crack, over a period of two to three days. The clay remained gently bound to the bases of the dishes, and could be rewetted by gently spraying the surface with a fine mist of deionized water, using a spray bottle held approximately $30 \mathrm{~cm}$ away from the clay surface. Water was added until the cracks in the center of the dish were seen to visibly close; a mass of water between 1 and 1.2 times the dried slurry mass was added.

Digital images of the clay surface were taken after each generation of drying. In some cases additional time-lapse images were taken throughout the course of drying. Permanent marks were made on the outside of each dish, which were used to align 
images in different generations. Typically these markers could be realigned to within one or two pixels, or an error of $0.1 \mathrm{~mm}$. The geometries of the resulting crack patterns were measured in these images. For most measurements the dominant error was due to sample size: there were large standard deviations in the observables, and each data set had approximately one hundred measurements. To avoid edge effects all cells either adjacent to, or one cell removed from, the walls of the dish were ignored. For layers thicker than $7 \mathrm{~mm}$ this did not leave enough remaining cells to report significant data. When studying the evolution of patterns over multiple generations, data were averaged over four separate dishes, in order to minimize statistical errors.

\section{Results}

\section{A. Crack formation}

When a clay layer was dried, rewetted, and then stirred to homogenize the slurry, the crack pattern in the next generation had no relation to the original pattern. Similarly, in layers that were rewetted until the clay was submerged, the subsequent crack pattern was largely unrelated to the original. Some similarities could be seen between generations, particularly in thicker layers, but most crack positions changed. If the clay was rewetted to the point where the cracks visibly closed, but the surface was not submerged, the crack pattern in the redried clay closely matched the pattern in the initially dried clay. However, this persistence was dependent on the layer thickness. For the thinnest layers, approximately $1 \mathrm{~mm}$ thick, rewetting the dried clay with a fine mist changed the positions of most cracks.

Time-lapse photos were taken every 5 min of a $2.9 \pm 0.1 \mathrm{~mm}$ thick drying clay layer, during the first 3 generations. In the first generation most cracks appeared between images. When cracks met, they formed $\mathrm{T}$-junctions, as a later crack will preferentially meet an earlier one in a normal direction. ${ }^{5}$ The cracks showed clear, well-defined edges, and the surface of the clay was smooth. After rewetting, cracks in later generations opened more slowly, often through the coalescence of several smaller cracks opening along some previous crack, and their faces become more uneven and jagged. Opening could take several minutes to occur, as observed in a few sequential images. After the first generation, the cracks formed shallow depressions in their vicinity, which remained visible in the rewetted layers. These depressions were less than a mm deep, but imply that the clay is of order $10 \%$ thinner in the vicinity of a previously open crack. The surface of the clay become rougher in later generations, but this was likely a result of the methods of rewetting the clay, which involved misting the surface with droplets of water. The changes in surface and crack roughness can be seen in Fig. 2 .

In each generation the pattern of cracks and vertices was similar, but the sequence in which cracks appeared was different. At any vertex there are three directions along which there are cracks. In each generation, at each vertex, one of these three cracks will open last. This choice was measured at all 243 vertices within an area, in the first and second generations of drying. At 159 vertices the sequence in which cracks appeared was the same in both generations. At 62 vertices, however, the sequence was different. In these cases, in the second generation, the crack approaching the vertex at approximately $90^{\circ}$ to its neighbors opened first, or second, rather than last. At the remaining 22 vertices, the sequence could not be determined. Thus, between $26 \%$ and $35 \%$ of the vertices formed through sequences of crack openings that were different in the first and second generations. If the uncertain vertices are assumed to be distributed with the same probabilities as the measured vertices, this percentage is estimated to be $28 \%$.

\section{B. Crack spacing}

Twenty-five dishes were prepared, each containing a clay slurry of different thickness. When dried, the resulting clay layers were between 1 and $7 \mathrm{~mm}$ thick. In most cases, the clay cracked into a network where each crack ended either on another crack, or on
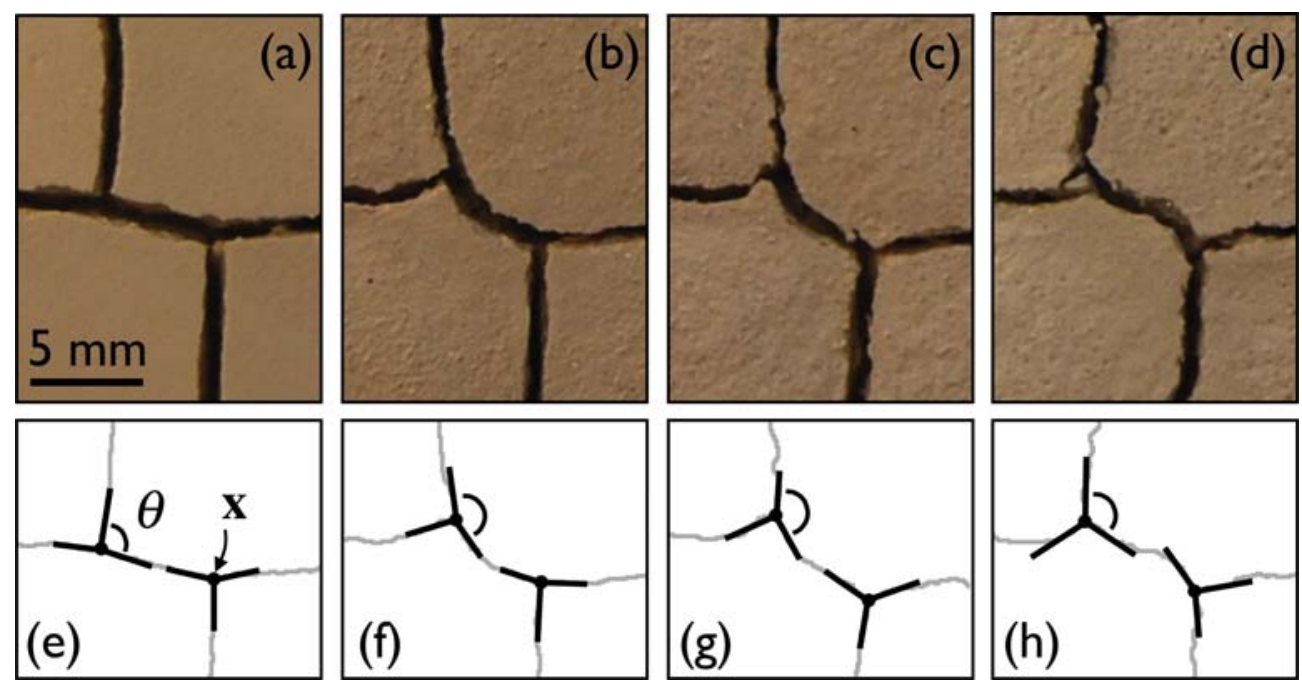

Fig. 2 Panels (a-d) show the evolution of two vertices in dried clay, after each of the first four generations of drying, respectively. There is a gradual change in fracture position, and an increase in the roughness of the crack with time. The vertices also move, such that all angles begin to approach $120^{\circ}$. This is highlighted in panels $(\mathrm{e}-\mathrm{h})$, which sketch out the positions of the same cracks, and demonstrate how the joint angles $\theta$ and vertex positions $\mathbf{x}$ are identified. 
the walls of the dish. These connected networks of cracks formed a tiling of the clay surface. For layers thinner than approximately $1.5 \mathrm{~mm}$, the cracks instead formed a disconnected network, where most cracks came to an end without intersecting another crack.

The average crack spacing was measured in two ways. First, the cross-sectional areas $A$ fully enclosed by cracks were measured, and the crack spacing was taken to be $\sqrt{ } A$. Second, the crack spacing was measured by randomly placing a line across a thresholded image of the crack pattern, and automatically detecting the distance between intersections of the line, and cracks. By averaging over many lines, a representative crack spacing was found. This method, the results of which are reported in Fig. 3, gives spacings consistently 10-15\% lower than the first method, but takes into account the contributions of unconnected cracks, and cracks which terminate within individual cells. The crack spacing increases with increasing layer thickness. This trend has been seen in diverse systems of dried slurries. ${ }^{2,3,6,7}$ The question of whether the crack spacing is directly proportional to the film thickness or merely increases with it remains open, and these data are not extensive enough to be definitive. However, crack spacings in thin layers, with unconnected cracks, appear to be larger than would be expected by a simple proportional relationship.

\section{Pattern dynamics}

The evolution of the crack pattern was observed in four clay samples, each $3.1 \pm 0.3 \mathrm{~mm}$ thick, over twenty-five consecutive drying, or generations. Layers of this thickness formed clearly observable crack patterns with over a hundred cells of dried clay, and could be rewetted without damaging the pattern. As can be seen in Fig. 4, all cracks in the pattern throughout these 25 generations can be identified in the original pattern. However, the fine details of the pattern are different in each generation. This is highlighted in Fig. 2, which shows how two crack vertices were modified through the first four generations.

Over multiple rewettings, the average area of the polygonal cells composing the dried crack patterns increased with time. In particular, the mean area increased by $20 \%$ within the first 5 generations, and retained this elevated value throughout the next 20 generations. This effect is due to the failure of some cracks to open in certain generations. It was not uncommon for a crack to

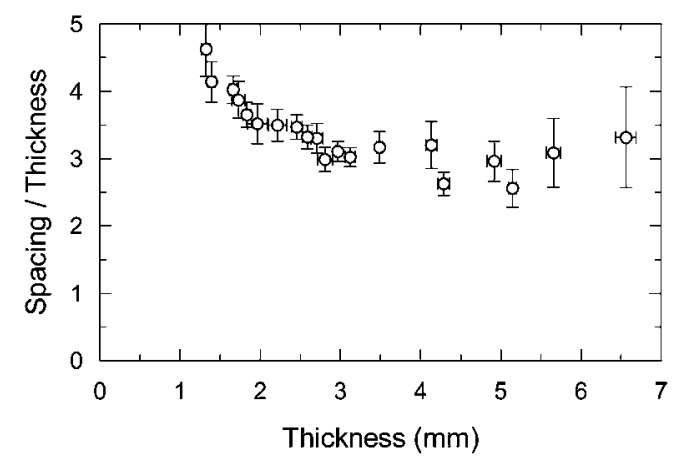

Fig. 3 The spacing of desiccation cracks in bentonite clay increases as the thickness of the cracking layer increases. For thin layers with unconnected cracks the crack spacing appears to be larger than expected from a simple proportional relationship.

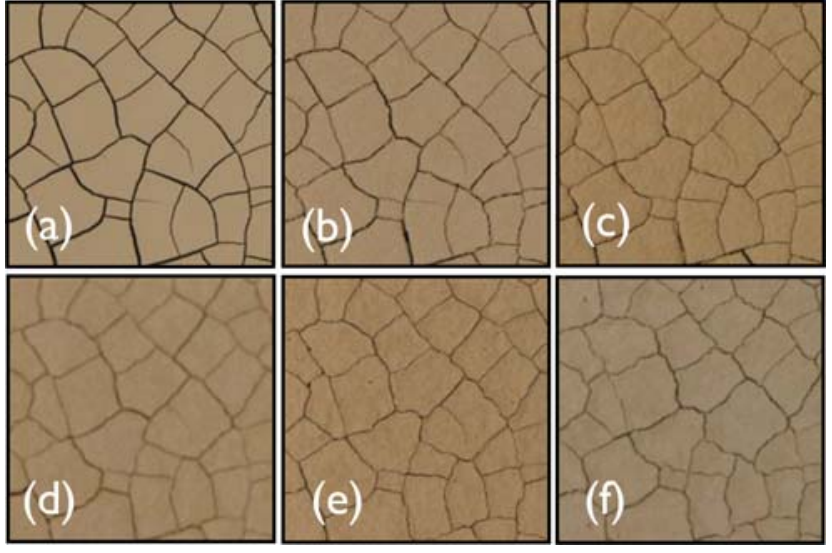

Fig. 4 The pattern of cracks changes slowly after many cycles of wetting and drying. The initial pattern (a) is dominated by cracks meeting at $90^{\circ}$ and $180^{\circ}$. Through generations (b) 3, (c) 5, (d) 10, (e) 15, and (f) 25 , the pattern slowly changes to one with many joints closer to $120^{\circ}$ angles. Each panel shows a $6 \times 6 \mathrm{~cm}^{2}$ area at the centre of a dried bentonite slurry.

be missing in one particular drying, only to reappear, in approximately its original place, in a later generation. Some cracks were seen to reappear up to ten generations after they had vanished, in this manner. When a crack vanishes, two adjacent cells merge into one, increasing the average area of the pattern. The distribution of areas does not otherwise change substantially between generations, as shown in Fig. 5. The standard deviation divided by the mean of the cell area, which describes the relative width of the area distribution, may decrease slightly over time, but this decrease is at most $10 \%$ over 25 generations.

In contrast to the relatively small changes in cell area, the angles between cracks at a vertex evolve rapidly, in these experiments. The joint angle, $\theta$, was defined as the angle between the tangent lines drawn on adjacent cracks, as they approached a vertex. These were measured by hand, taking care to measure the tangent directions as close to the vertex as possible, although the finite width of the cracks necessitated averaging over the 1-2 mm of crack closest to the vertex. This method is demonstrated in Fig. 2. For each of the four samples, between 100 and 200 angles were measured in different drying generations.

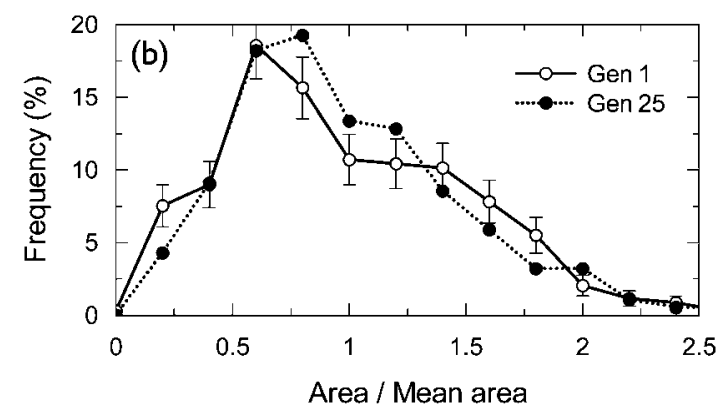

Fig. 5 The average cross-sectional area of clay cells increases slightly in the first 5 generations, as some cracks will not be open in any particular generation. However, histograms of the distributions of cell areas do not show any other substantial differences between the 1st and 25 th generations. Representative error bars are shown for Generation 1. 


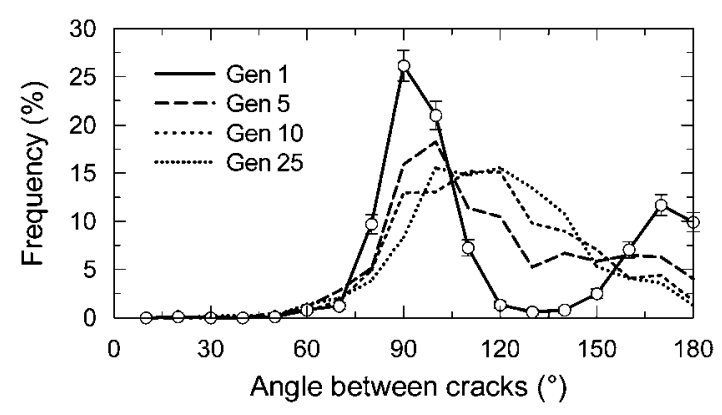

Fig. 6 The distribution of joint angles in clay layers changes with repeated cycles of wetting and drying. The distribution is peaked at $90^{\circ}$ and $180^{\circ}$ in the initial pattern, but tends towards $120^{\circ}$ after repeated dryings. Representative error bars are shown on Generation 1, and all data are averaged over $10^{\circ}$ bins.

Initially, as shown in Fig. 6, most vertices contain cracks at $90^{\circ}$ and $180^{\circ}$ to each other. In subsequent generations, this distribution of angles shifts, and the two peaks of the distribution of angles approach each other. After only 10 generations, the distribution is broadly centered around a single peak at $120^{\circ}$, as shown in Fig. 6.

As joint angles change, it can become difficult to distinguish between T-junctions and Y-junctions. Since the average angle of three cracks meeting at a vertex is always $120^{\circ}$, the standard deviation

$$
\sigma^{2}=\frac{1}{N} \sum_{i=1}^{N}\left(\theta_{i}-120^{\circ}\right)^{2}
$$

was used to characterize the relaxation of joint angles $\theta$. The standard deviation decreases consistently throughout the first ten generations, after which time it ceases to change noticeably. A final value of $\sigma_{o}=24.9 \pm 0.5^{\circ}$ describes generations 15 through 25 . The timescale over which the angles relaxed was measured by fitting an exponential decay to the evolution of $\sigma$, as shown in Fig. 7. A decay time of $4.2 \pm 0.5$ generations was found. If the percentage of joint angles in the range of $105^{\circ}$ to $150^{\circ}$ is instead taken to be representative of the proportion of $\mathrm{Y}$-junctions, as shown in Fig. 7(c), a similar relaxation is found, with a decay time of $3.7 \pm 0.6$ generations.

As the cracks around a vertex change, the vertex also moves. Absolute changes in vertex positions $\mathbf{x}$ were measured by comparing images of crack patterns. The average displacement

$$
\delta=\frac{1}{N} \sum_{i=1}^{N}\left|\mathbf{x}_{0 i}-\mathbf{x}_{i}\right|
$$

was calculated in different generations, where $\mathbf{x}_{0}$ are the vertex positions after the first drying. As shown in Fig. 8(a), there is a substantial change in the vertex positions between the first and second generations, followed by a more gradual, but continuous, motion. In these experiments, there is an average movement of each vertex, between generations 2 and 25 , of $30 \mu \mathrm{m}$ per generation. It is interesting to note that this motion continues after the crack angles have stabilized. This suggests that there may be dynamics that continue well past the 25 generations observed in this study. After 25 generations, vertices had shifted their positions, on average, by slightly more than $1 \mathrm{~mm}$, or approximately $10 \%$ of the average fracture spacing.

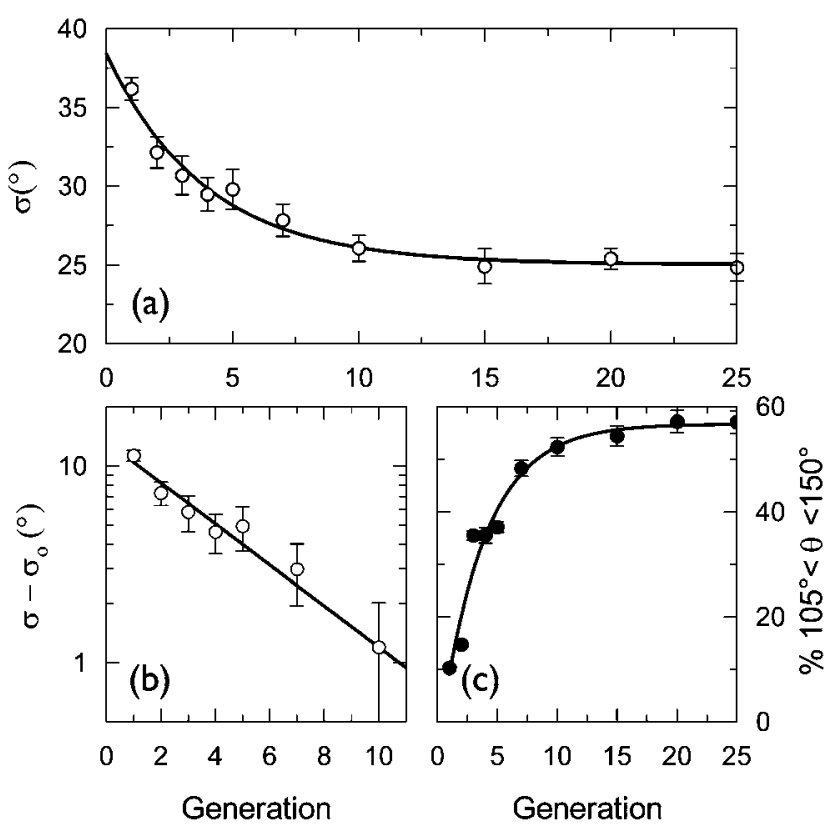

Fig. 7 (a) The standard deviation of joint angles, $\sigma$, decreases rapidly within the first 10 generations, to a final value $\sigma_{o}$. (b) An exponential decrease towards $\sigma_{o}$ is well-fitted to the data, with a decay time of $4.2 \pm$ 0.5 generations. (c) The proportion of angles close to $120^{\circ}$ also shows an exponential relaxation, with a decay time of $3.7 \pm 0.6$ generations.

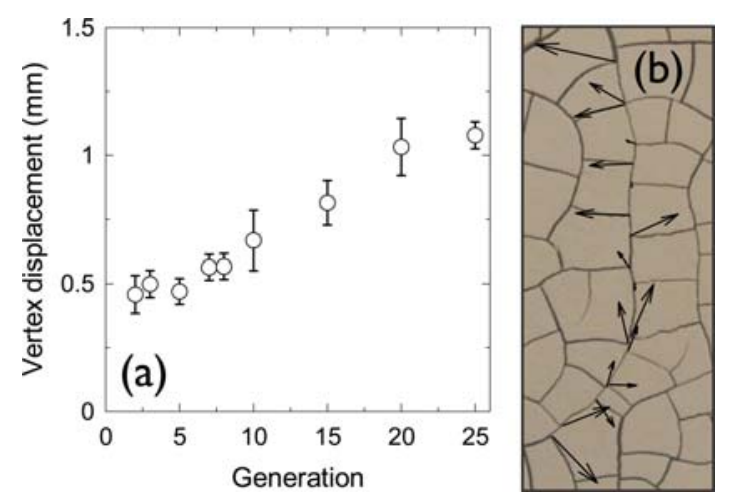

Fig. 8 (a) Direct measurements of vertex displacement, relative to the pattern in generation 1 , show a large change in generation 2 , followed by a continuous motion away from the original location. (b) The individual motion of vertices, shown as vectors representing the change in position between generations 1 and 25, has a weak tendency to follow the direction of the crack originally branching from the vertex at two right angles.

The motion of a vertex has a weak tendency to be normal to the original primary crack. In each experiment, a single primary crack was chosen. This crack opened early enough in the first generation so that all adjoining cracks intersect it at right angles. Correlations of the change in vertex positions between subsequent generations could not be detected to within experimental uncertainty. However, the positions of the vertices along the crack were compared after 1 and 25 cycles of wetting and drying. In each of the four dishes, $70 \pm 10 \%$ of the vertices were observed to move in a direction on the same side of the crack approaching the vertex at right angles. This is demonstrated in Fig. 8(b). 


\section{Discussion}

The experiments presented here show that crack patterns in clay evolve when the clay is repeatedly wetted and dried. Over approximately ten generations the distribution of joint angles shifted from being peaked at $90^{\circ}$ and $180^{\circ}$ to having a single peak at $120^{\circ}$. This relaxation was accompanied by a gradual movement of the vertices of the crack pattern. We found that the displacement $\delta$ of a vertex from its original position increased slowly in successive generations, up to at least twenty-five generations. Where three cracks met at a T-junction, this motion tended to shorten the crack that had originally approached a straight crack at two right angles. Further work would be required to determine whether this motion is ballistic $(\delta \sim m$ after $m$ rewettings), Brownian $\left(\delta \sim m^{1 / 2}\right)$, or a mixture of the two.

It is likely that the equilibrium state was not fully reached in this experiment, as we were still observing directed changes in the vertex positions in generation 25 . This suggests that there may be additional interesting long-term evolution to a mud-crack pattern, including, for example, a more gradual narrowing of the cell area or joint angle distribution.

Here, we argue that the evolution of crack patterns from rectilinear T-junctions towards hexagonal Y-junctions follows from three aspects of crack behavior. First, that it is easier to crack along the path of a previously open crack. Second, that a crack will advance in a way to relieve the most local strain energy possible, whilst minimizing the energy cost in creating new crack faces. Finally, that each time the layer cracks, the sequence of crack opening may be different.

The crack patterns reported here show a long-term persistence in the position of cracks, coupled with gradual pattern dynamics. Information about crack positions must be stored in the clay layer, across multiple wettings and dryings. A similar concept was demonstrated by Nakahara and Matsuo, ${ }^{8}$ who vibrated slurries prior to drying them. The resulting cracks were largely co-aligned with the direction of vibration. There are, however, more concrete features in our experiments that could also lead to the persistence of the crack positions. The cracks may not heal completely, especially at depth, as the clay is not completely resaturated. Under such conditions, the positions of previous cracks would be weaker than the surrounding clay. Also, the shallow depressions that form around cracks cause the layer thickness to be thinner near old crack locations. Opening a crack in an area which is weakened or thinned by a partially healed crack requires less work.

A rectilinear crack pattern provides information regarding the sequence in which cracks formed. ${ }^{5}$ However, this sequence differs across generations. When a crack approaches the position of a vertex, prior to the opening of any other cracks in the vicinity, there is a choice presented. The crack can halt at the vertex, it can continue to propagate down one, or the other, leg of the vertex, or it can split and propagate down both remaining legs of the vertex.

Consider the case where a propagating crack changes direction near the position of a vertex from the previous generation. Perhaps it approaches along what had been the secondary crack, and continues along the path of one of the primary cracks. In a homogeneous medium, the crack will tend to open in a way to maximize the difference between the strain energy released, and the fracture energy spent. ${ }^{9}$ This fracture energy, or a corresponding fracture toughness, has been well characterized for a variety of clays and soils. ${ }^{10,11}$ As the crack approaches the vertex, the total crack length will be less if it curves around the vertex, rather than kinking. By bending, however, the crack will go through stiffer material. The total fracture energy will therefore represent a balance between these effects. When the saving in energy is sufficient, the crack should round the vertex, as sketched in Fig. 9(a).

If a single change in the sequence of crack formation were to modify a T-junction into a Y-junction, and this sequence has a probability $p$ of changing in each generation, then the number of T-junctions in the crack pattern would decrease exponentially, with a fraction

$$
n_{T}=(1-p)^{m}=e^{-m / \tau}
$$

remaining after $m$ rewettings. In the experiments presented here, between the first two generations, it was found that $p=0.28 \pm$ 0.04 . Under these conditions, one would expect a decay time of $\tau=-1 / \ln (0.72)=3.0 \pm 0.5$ generations. Indeed, the observed distributions of joint angles appear to relax exponentially, as was shown in Fig. 7. Two methods were used to characterize the evolution of T-junctions to Y-junctions, with measured decay times of $4.2 \pm 0.5$ and $3.7 \pm 0.6$ generations, respectively. These generally agree with, but are slightly slower than, the prediction just made. It should be noted that it may take more than one change in crack sequence to fully adjust the vertex and relax the joint angles.

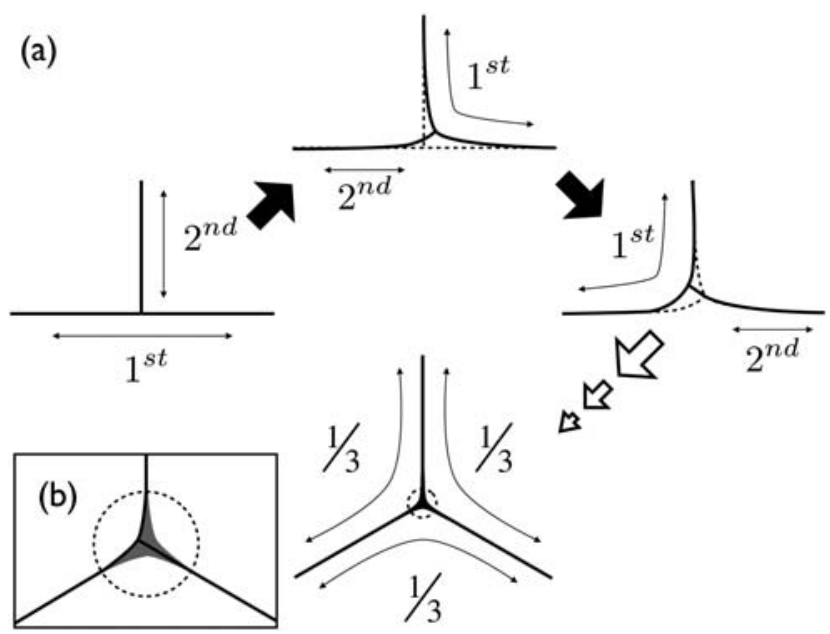

Fig. 9 The crack pattern in a clay layer changes when the layer is repeatedly wetted and dried. (a) A T-junction (left) contains information about the order of crack formation, as a later crack will intersect an earlier crack at right angles. In different generations (progressing clockwise), the order of cracks can differ, encouraging adjustments in the crack pattern. After many generations (bottom), if each crack path is equally likely to form first, symmetry suggests a Y-junction. (b) Very close to the vertex of a Y-junction, the cracks may still curve, in response to the order of crack formation in any particular generation. Over all possible paths, these changes will cancel out for a Y-junction. However, for a T-junction, the vertex will move, on average, as indicated in (a). Compare the process sketched here to that observed in Fig. 2. 
After many generations, a Y-junction should be stable to random changes in the sequence of crack formation. As shown in Fig. 9(a), if each crack path opens first with equal frequency, then there is no average tendency for the vertex of a Y-junction to move in any direction. In each particular generation, the path taken by the initial crack may still cause some curvature near the vertex, as shown in Fig. 9(b), but these perturbations will cancel out on average, maintaining the shape of the Y-junction.

In an outdoor setting, cycles in the weather cause soil to be repeatedly wetted and dried, providing the conditions for mud cracks to evolve towards $120^{\circ}$ joint angles. This environment is unlikely to be as controlled, or gentle, as our rewetting procedure. In compensation, the crack patterns in some soils may be more persistent than these experiments suggest. The hexagonal pattern shown in Fig. 1(c) is of Racetrack Playa, in Death Valley, which is periodically inundated with water. Similarly, areas near the pattern shown in Fig. 1(d) were visibly submerged, yet hexagonal.

The experimental conditions used in this study are also somewhat analogous to those of polygonal terrain, which forms in repeatedly frozen and thawed soils. There, each annual thermal cycle contains one generation of fracture. ${ }^{12,13}$ Over many such cycles, it is thought that an initially rectilinear pattern of cracks evolves towards a more hexagonal pattern, ${ }^{14}$ although, due to the timescales involved, this has never been directly observed. The work reported here clearly demonstrates the plausibility of such an effect.

\section{Conclusion}

Mud-crack patterns found in nature often appear to have hexagonal order, and be composed of Y-junctions with $120^{\circ}$ angles. This is in contrast to the rectilinear craquelure patterns, dominated by $\mathrm{T}$-junctions, that have more generally been studied experimentally. The results presented in this paper show that if a cracked clay layer is repeatedly wetted and dried, the crack pattern slowly evolves from a rectilinear pattern towards a hexagonal one. In a clay layer of a few mm thick, this happens in only 5 to 10 generations of drying and wetting. This relaxation is accompanied by all joint angles approaching $120^{\circ}$, and by a gradual motion of the crack vertices.

\section{Acknowledgements}

LG wishes to thank Ron Sletten, Bernard Hallet, Dan Mann and Birgit Hagedorn for fruitful discussions, and B. Hallet additionally for allowing the use of his images of mud cracks. Funding from the Engineering and Physical Sciences Research Council [EP/E05949X/1] is gratefully acknowledged.

\section{References}

1 A. Corte and A. Higashi, US Army Snow Ice and Permafrost Research Report, 1960, vol. 66, 48pp.

2 K. Pasricha, U. Wad, R. Pasricha and S. Ogale, Phys. A, 2009, 388, $1352-1358$.

3 K. A. Shorlin, J. R. de Bruyn, M. Graham and S. W. Morris, Phys. Rev. E: Stat. Phys., Plasmas, Fluids, Relat. Interdiscip. Top., 2000, 61, 6950 .

4 G. Müller, J. Struct. Geol., 2001, 23, 45.

5 S. Bohn, L. Pauchard and Y. Couder, Phys. Rev. E: Stat., Nonlinear, Soft Matter Phys., 2005, 71, 046214.

6 S. Bohn, J. Platkiewicz, B. Andreotti, M. Adda-Bedia and Y. Couder, Phys. Rev. E: Stat., Nonlinear, Soft Matter Phys., 2005, 71, 046215.

7 A. Groisman and E. Kaplan, Europhys. Lett., 1994, 25, 415-20.

8 A. Nakahara and Y. Matsuo, J. Phys. Soc. Jpn., 2005, 74, 1362-1365.

9 B. Cotterell and J. R. Rice, Int. J. Fract., 1980, 16, 155-169.

10 F.-H. Lee, K.-W. Lo and S.-L. Lee, J. Geotech. Eng., 1988, 114, 915929.

11 J.-J. Wang, J.-G. Zhu, C. F. Chiu and H. Zhang, Eng. Geol., 2007, 94, 65-75.

12 A. L. Washburn, Geol. Soc. Am. Bull., 1956, 67, 823-865.

13 A. H. Lachenbruch, U.S. Geol. Surv. Spec. Paper, 1962, 70, 69.

14 R. S. Sletten, B. Hallet and R. C. Fletcher, J. Geophys. Res., 2003, 108, 8044 . 